\title{
The Golden Age of Cataclysmic Variables and Related Objects (Old and News)
}

\author{
F. Giovannelli ${ }^{1}$, L. Sabau-Graziati ${ }^{2}$ \\ ${ }^{1}$ INAF - Istituto di Astrofisica e Planetologia Spaziali, Area di Ricerca di Tor Vergata, Via Fosso del Cavaliere, 100 - \\ I0017r Roma, Italy \\ ${ }^{2}$ INTA-Dpt. Cargas Utiles y Ciencias del Espacio, C/ra de Ajalvir, Km 4 - E28850 Torrejón de Ardoz, Madrid, Spain \\ Corresponding author: franco.giovannelli@iaps.inaf.it
}

\begin{abstract}
In this paper we review cataclysmic variables $(\mathrm{CVs})$ discussing several hot points about the renewing interest of today astrophysics about these sources. We will briefly discuss also about classical and recurrent novae, as well as the intriguing problem of progenitors of the Type Ia supernovae. This paper is an extended and updated version of the review by Giovannelli (2008). Because of limited length of the paper and our knowledge, this review does not pretend to be complete. However, we would like to demonstrate that the improvement on knowledge of the physics of our Universe is strictly related also with the multifrequency behaviour of CVs, which apparently in the recent past lost to have a leading position in modern astrophysics.
\end{abstract}

Keywords: cataclysmic variables - dwarf novae - intermediate polars - polars - novae - optical - spectroscopy - photometry - sub-mm - IR - radio - UV - X-rays - multifrequency astrophysics - X-ray binary systems - high energy astrophysics.

\section{Introduction}

In the 1950s it was recognized that the various phenomena displayed by the CVs are all the consequence of accretion of matter onto a white dwarf (WD) from a low mass donor star (e.g., Warner, 1976; 1995a). CVs are binary systems in which the primary component is a WD $\left(M_{\mathrm{wd}} \sim 1 \mathrm{M}_{\odot}\right)$ and the secondary is a late type Main Sequence star $\left(M_{\mathrm{s}} \leq 1 \mathrm{M}_{\odot}\right)$ (e.g., Smak, 1985a).

Mass transfer is strongly depending, besides the orbital parameter of the system, on the magnetic field intensity at the surface of the primary. Such process produces a large fan of behaviour that are detectable in different energy ranges: from radio to X-rays, and even in $\gamma$-rays. The orbital periods of $\mathrm{CVs}$ are ranging from $\sim 80 \mathrm{~m}$ to $\sim 12 \mathrm{~h}$ with a distribution showing a gap between 2 and 3 hours, in which few systems have been detected. In the past this gap was empty and this was the reason because was nicknamed 'period gap'.

More than $600 \mathrm{CVs}$ are known, most of them discovered through optical observations, and some, especially those in which the magnetic field of the WD is strong, discovered through X-ray observations, but with the detectors of the second and further generations, since CVs are in general not very bright in X-ray energy range. Those with known or suspected orbital period are listed by Ritter \& Kolb (1998).

The first CV detected in the X-ray range, with rocket experiments, was the dwarf nova SS Cyg (Rappaport et al., 1974; Heise et al., 1978). The UHURU satellite detected two CVs, which were not recognized as such. Warner (1976) proposed the identification of $4 \mathrm{U}$ 1249-28 with EX Hya, and the variable AM Her, which on further optical studies was recognized as a CV (Forman et al., 1978). The magnetic field in these two systems is strong $\left(\approx 10^{7}-10^{8} \mathrm{G}\right)$. A few dozen CVs were detected in X-rays with HEAO-1 satellite, with EXOSAT, and with the Einstein satellite (e.g., reviews of Cordova \& Mason, 1983; Cordova, 1995). Later Verbunt et al. (1997) recognized $91 \mathrm{CVs}$ from a sample of 162 systems with known or suspected binary periods by using data of the ROSAT XRT-PSPC All Sky Survey.

Historically, because CVs were observed photometrically and without seeming to follow any regular pattern, they were named with the term cataclysmic (from the Greek word kataklysmos = flood, storm; Hack \& la Dous, 1993). As collecting of observational data progressed it became apparent that these objects were regular binary systems which for some reason changed in brightness; some of them also regularly (Recurrent Novae and Dwarf Novae) while some others only once (Classical Novae). Therefore the classification of CVs was based on the optical outburst properties, by which one may distinguish four groups of CVs: (i) classical novae; (ii) recurrent novae; (iii) dwarf novae; (iv) nova- 
like objects (e.g., Giovannelli \& Martinez-Pais, 1991 and references therein; Ritter, 1992; Giovannelli, 2008). This classification, however, is neither self-consistent nor adequate and it is much better to consider primarily the observed accretion behaviour (Smak 1985b). One obvious advantage of such an approach is connected with the time scales of various accretion phenomena, which are sufficiently short to avoid any major observational bias: the mass accretion rates in CVs usually range from $10^{-11}$ to $10^{-8} \mathrm{M}_{\odot} \mathrm{yr}^{-1}$ (Patterson, 1984); the time scales are from tens of seconds (oscillations in dwarf novae at outbursts) to years (super-outbursts of SU UMa stars or long term variations in VY Scl stars).

However, in the class of nova-like objects there are two sub-classes: the DQ Her stars and the AM Her stars. In these sub-classes of CVs the WDs possess magnetic fields with intensity enough high for dominating the accretion disk and all the phenomena related to it. These classes of magnetic CVs, whose names are coming from the prototypes DQ Her and AM Her took later the names of Intermediate Polars and Polars, respectively. A short history of their discovery has been discussed by Warner (1995b). Fundamental papers about these subclasses are those by Patterson (1994), Warner (1996). The class of IPs has been split into two subclasses with relatively large and relatively weak magnetic field (Norton et al., 1999). One example of a system belonging to the latter subclass is DO Dra (previously registered as YY Dra) (Andronov et al., 2008).

There is another class of CVs, the rare AM Canum Venaticorum (AM CVn) star systems. They have extremely short orbital periods between $\sim 10-65$ minutes. Their spectra do not show evidence for hydrogen. They appear to be helium-rich versions of CVs (e.g. Warner, 1995c; Nelemans, 2005). There is an old suggestion, that in these systems the mass transfer is driven by gravitational wave radiation losses, proposed by Paczyński (1967), after the discovery of the prototype with an orbital period of $\sim 17$ minutes (Smak, 1967).

Depending on the magnetic field intensity at the WD, the accretion of matter from the secondary star onto the primary can occur either via an accretion disc (in the so-called Non-Magnetic CVs: NMCVs) or a channelling through the magnetic poles (in the case of Polars: PCVs) or in an intermediate way (in the case of Intermediate Polars: IPCVs).

$\mathrm{CVs}$ in a time scale of order between weeks and years flare up almost periodically, about few magnitudes in optical wavelengths; the duration of the outbursts is much shorter than the recurrence time. Typical light curves for classical novae and dwarf novae of the U Gem, Z Cam, and SU UMa types can be seen in Ritter (1992) and e.g. in Giovannelli (2008).

The recurrence time-scale of outbursts in dwarf no- vae is correlated with their amplitude and the outburst duration is depending on the orbital period (Warner, 1987).

In PCVs the WD magnetic field is strong enough to make the Alfvén radius greater than the circularization radius, so no accretion disc is formed and the accretion structure is fully governed by the magnetic field, which canalize the accreting matter across the field lines. Owing to the intense magnetic field $(\sim 10-200 \mathrm{MG})$, the WD rotation is synchronized with the binary orbital period (a few hours). However, there are few systems (V1432 Aql, BY Cam, V1500 Cyg, V4633 Sgr, and CD Ind) in which $\mathrm{P}_{\text {spin }}$ and $\mathrm{P}_{\text {orb }}$ differ by around $2 \%$ or less. These are assumed to be polars that have been disturbed from synchronism by a recent nova explosion (Norton, Sommerscales \& Wynn, 2004, and the references therein).

IPCV WDs have moderate magnetic fields (order of a few MG); the Alfvén radius is smaller than the circularization radius but it is greater than the WD radius. Therefore an accretion disc is formed in these systems but being disrupted at its inner region. In IPCVs matter follows again the magnetic field lines but just inside the Alfvén radius. The rotating WD is asynchronous with the binary orbital period $\left(\mathrm{P}_{\text {spin }} \ll\right.$ $\left.\mathrm{P}_{\text {orb}}\right)$. However, there are few systems that may be best described as nearly synchronous intermediate polars (V381 Vel, RXJ0524+42, HS0922+1333, and V697 Sco) (Norton, Sommerscales \& Wynn, 2004, and the references therein). Two of these systems lie in the 'period gap'. Probably all four systems are IPs in the process of attaining synchronism and evolving into polars.

The last group defined by the accretion structure criterion, NMCVs, includes those systems whose WD magnetic fields are not relevant in governing the accretion structure. In these systems the accretion disc extends down to the WD surface and a boundary layer is formed. This family shows a great diversity of observational behaviour; for this reason the historical criterion of classification is, in this case, more appropriate for distinguishing their sub-classes. However, it is simply an attempt of classification for lack of a more general physical classification (e.g., Giovannelli, 1991 and references therein). Indeed, in general, we can consider the WD of a $\mathrm{CV}$ as a gravimagnetic rotator, characterized by a mass $M$ - accreting matter at a rate $\dot{M}$ from the optical companion (the secondary star) — rotating with a velocity $\vec{\omega}$ and having a magnetic moment $\vec{\mu}$, not necessarily coaxial with the rotational axis (Lipunov, 1987; 1991). Then the accreting system is completely characterized by the following physical parameters: mass $\mathrm{M}$, accretion rate $\dot{\mathrm{M}}$, rotational velocity $\vec{\omega}$, and magnetic moment $\vec{\mu}$. In the plane spin period of the WD (and in general of the compact object) - gravimagnetic param- 
The Golden Age of Cataclysmic Variables and Related Objects (Old and News)

eter $\mathrm{y}=\dot{\mathrm{M}} / \mu^{2}$ it is possible to find any sort of physical conditions of gravimagnetic rotators, as discussed by e.g. Giovannelli (1991).

Figure 1 shows the positions of few CVs in the diagram spin period of the WD - gravimagnetic parameter (in unit -42): AM Her (Terada et al., 2010 and references therein), AE Aqr (Patterson, 1979; de Jager et al., 1994; Wynn, King \& Horne, 1997), DQ Her (Patterson, 1994; Zhang et al., 1995), EI UMa (Reimer et al., 2008), SS Cyg (Giovannelli \& Sabau-Graziati 2012a). It appears evident the power of this diagram obtained by Lipunov (1987) using $1 \mathrm{M}_{\odot}$ white dwarf. The polars AM Her and AE Aqr lie in the zone of propeller, how they must stay, while the IPCV DQ Her (the prototype of this class), EI UMa (a very well known IPCV), and SS Cyg (whose nature as IPCV is claimed by Giovannelli's group on the base of many circumstantial proofs and also because of a cogent similarity with EI UMa, e.g. Giovannelli \& Sabau-Graziati 2012a,c) lie just in the zone predicted by Lipunov for such objects.

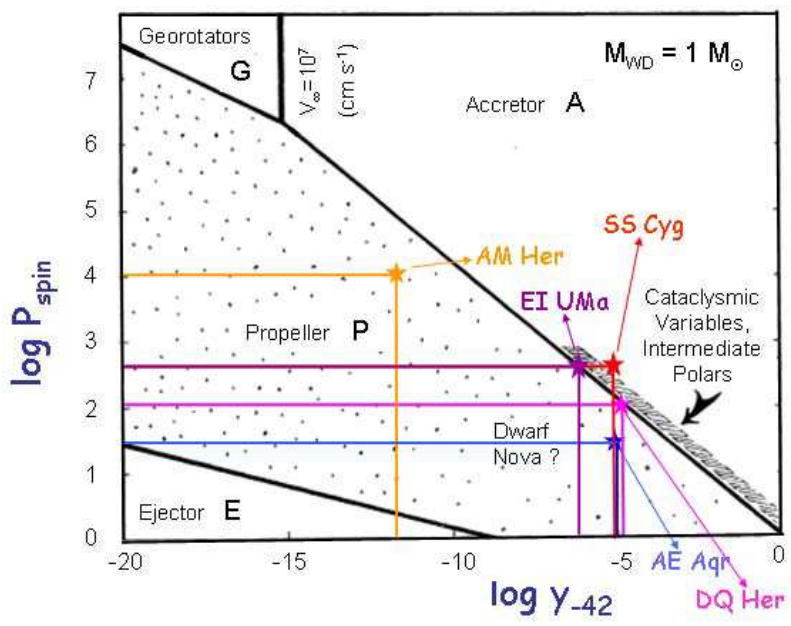

Figure 1: The positions of several CVs in Lipunov's diagram calculated for $1 \mathrm{M}_{\odot}$ white dwarf (after Lipunov, 1987).

As recalled by Giovannelli \& Sabau-Graziati (1999), it is evident that the properties of an outburst in CVs depend crucially on the accretion rate, the mass of the $\mathrm{WD}$, and the chemical composition of its hydrogen rich envelope in which the thermonuclear runaway occurs. And the accretion process onto the WD is strongly influenced by its magnetic field intensity. Indeed, the three kind of CVs (non-magnetic, polars, and intermediate polars) obey to relationships between the orbital period of the system and the spin period of the WD (Warner \& Wickramasinghe, 1991), where the magnetic field intensity plays a fundamental role. The orbital evolution of CVs, and hence the mass-transfer rate $(\dot{\mathrm{M}})$ from the secondary to the white dwarf is driven by mag- netic braking of the secondary for long-period systems $\left(\mathrm{P}_{\text {orb }}>3 \mathrm{hr}\right)$ and gravitational radiation for shortperiod systems $\left(\mathrm{P}_{\text {orb }}<2 \mathrm{hr}\right)$.

However, such a gap - which was believed true for long time - is now partially filled by the SW Sex systems (e.g. Rodriguez-Gil, 2003; Rodriguez-Gil et al., 2007). The apparent 'period gap' was due to a smaller number of systems having orbital periods in such an interval, which were escaping from the observations.

Therefore the investigation on the magnetic field intensities in WDs is crucial in understanding the evolution of CVs systems. The fundamental parameters to be searched are the magnetic moment, the mass accretion rate and the orbital parameters of the systems. In this way it will be possible to fulfill the plane $\log \mathrm{P}_{\text {spin }}{ }^{-}$ $\log \mathrm{P}_{\text {orb }}$, where a priori there are not restricted ranges of magnetic moment $|\vec{\mu}|$, or special correlations between $\mathrm{P}_{\text {spin }}$ and $\mathrm{P}_{\text {orb }}$ and $|\vec{\mu}|$. The distribution of objects in that diagram is owed to the interaction of braking torques and accretion torques, with the superposition of the observed or implied variations of the accretion rate on long time scale ( $>10^{2} \mathrm{yr}$ ), acting on a continuum of magnetic moments. In this way each system is completely described by those physical parameters.

Davis et al. (2008) applied population synthesis techniques to calculate the present day number of two types of WD-main sequence star (WDMS) binaries within the 'period gap'. The first are post-common envelope binaries with secondary stars that have masses $0.17 \leq \mathrm{M}_{\mathrm{s}} / \mathrm{M}_{\odot} \leq 0.36$ (gPCEBs), such that they will commence mass transfer within the period gap. The second type are systems that were CVs at some point in their past, but detached once they evolved down in orbital period to $\approx 3 \mathrm{~h}$ as a consequence of disrupted magnetic braking, and are crossing the 'period gap' via gravitational radiation $(\mathrm{dCVs})$. They predicted an excess of dCVs over gPCEBs within the 'period gap' of $\sim 4$ to $\sim 13$. This excess is revealed as a prominent peak at the location of the 'period gap' in the orbital period distribution of the combined gPCEB and dCV population. They suggest that if such a feature is observed in the orbital period distribution of an observed sample of short orbital period WDMS binaries, this would strongly corroborate the disruption of magnetic braking.

Willems et al. (2005) and Willems et al. (2007) by using population synthesis tools studied the population of NMCVs with orbital periods 1$)<2.75 \mathrm{~h}$, and 2 ) $>2.75 \mathrm{~h}$, respectively.

1) A grid of detailed binary evolutionary sequences was calculated and included in the simulations to take account of additional angular momentum losses beyond that associated with gravitational radiation and mass loss, due to nova outbursts, from the system. As a specific example, Willems et al. (2005) considered the 
effect of a circumbinary disc to gain insight into the ingredients necessary to reproduce the observed orbital period distribution. The resulting distributions showed that the period minimum lies at about 80 minutes, with the number of systems monotonically increasing with increasing orbital period to a maximum near 90 minutes. There is no evidence for an accumulation of systems at the period minimum, which is a common feature of simulations in which only gravitational radiation losses are considered. The shift of the peak to about 90 minutes is a direct result of the inclusion of systems formed within the period gap.

2) The population of NMCVs with unevolved mainsequence-like donors at orbital periods greater than $2.75 \mathrm{~h}$ was investigated. In addition to the angular momentum losses associated with gravitational radiation, magnetic braking, and mass loss from the system, Willems et al. (2007) also included the effects of circumbinary discs on the evolution. For a fractional mass input rate into the disc, corresponding to $3 \times 10^{-4}$ of the mass transfer rate, the model systems exhibit a bounce at orbital periods greater than $2.75 \mathrm{hr}$. The simulations revealed that: i) some systems can exist as dwarf novae throughout their lifetime, ii) dwarf novae can evolve into novalike systems, and iii) novalike systems can evolve back into dwarf novae during their postbounce evolution to longer orbital periods. Among these subclasses, novalike cataclysmic variables would be the best candidates to search for circumbinary discs at wavelengths $\geq 10 \mu \mathrm{m}$. The theoretical orbital period distribution is in reasonable accord with the combined population of dwarf novae and novalike systems above the period gap, suggesting the possibility that systems with unevolved donors need not detach and evolve below the period gap as in the disrupted magnetic braking model. Experimental data are necessary for checking the validity of theoretical predictions.

The field strength distribution of MCVs differs from that of single WDs, although both cluster around 30 MG. The distribution of isolated WDs extend on a wide range of magnetic field strength $\left(\sim 10^{5}-10^{9} \mathrm{G}\right)$, whilst in accreting WDs of CVs, as far as is presently known, there is a lack of systems at both high and low field strengths. However, the apparent absence of low field MCVs might be explained by the IPs, which generally have unknown field strengths, and the lack of high field systems is still not understood (e.g., Beuermann, 1998). Wynn (2000) discussed the problem of accretion flows in MCVs. On the base of the ratio $\mathrm{P}_{\text {spin }} / \mathrm{P}_{\text {orb }}$ he divided the MCVs in three classes: class 1, class 2 and class 3 if such a ratio is $\ll 0.1, \sim 0.1$, and $\gg 0.1$, respectively. For the systems in class 1 the disc equilibrium condition is clearly satisfied. Those in class 2 are very unlikely to possess accretion discs. The systems in class 3 are EX Hya-like systems which lie below the 'period gap' and cannot possibly contain accretion discs. These are EX Hya, HT Cam, RXJ1039.7-0507, and V1025 Cen. These all have $\mathrm{P}_{\text {spin }} / \mathrm{P}_{\text {orb }}>0.1$ and $\mathrm{P}_{\text {orb }}<2$ hr. DD Cir and V795 Her lie within the 'period gap' with $\mathrm{P}_{\text {spin }} / \mathrm{P}_{\text {orb }} \sim 0.1$ and may be included in the class 2 (Norton, Somerscales \& Wynn, 2004, and the references therein). Wynn (2000) crudely classified the MCVs according to the magnetic moment and orbital period. EX Hya systems have magnetic moment similar to IPs above the 'period gap' and comparable to the weakest field AM Her-like systems. This indicates that MCVs above the 'period gap' will evolve to long spin periods below it. Norton, Wynn \& Somerscales (2004) investigate the rotational equilibria of MCVs. They predict that IPCVs with $\mu \geq 5 \times 10^{33} \mathrm{G} \mathrm{cm}^{3}$ and $\mathrm{P}_{\text {orb }}>3 \mathrm{hr}$ will evolve into $\mathrm{PCV}$, whilst those with $\mu \leq 5 \times 10^{33} \mathrm{G} \mathrm{cm}^{3}$ and $\mathrm{P}_{\text {orb }}>3 \mathrm{hr}$ will either evolve into low field strength polars that are presumably unobservable, and possibly EUV emitters, or into PCVs when their fields, buried by high accretion rate, revive when the mass accretion rate reduces.

Warner (1996) deeply discussed torques and instabilities in IPs on the base of measured spin periods of the primaries and found several important relationships between fundamental parameters of these systems, such as $\log \dot{M}$ vs $\log \mathrm{P}_{\text {orb }}, \log \mu_{33}$ vs $\log \dot{\mathrm{M}}_{17}, \log \mathrm{L}_{\mathrm{X}}$ vs $\log \dot{\mathrm{M}}$, as shown in Fig. 2 in the left, central, and right panels, respectively. There is a range of magnetic moments $\mu$ and mass transfer rates in which synchronized rotation of the primary can occur even though it possesses an accretion disc.

Ak et al. (2010), using available astrometric and radial velocity data, computed the space velocities of CVs with respect to the Sun and investigated kinematical properties of various sub-groups of CVs. The orbital period distribution of CVs in the refined sample of 159 systems resembles that of the whole sample of CVs (e.g. Connon Smith, 2007). Ak et al. (2010) found that the mean kinematical age (MKA) of the 159 systems is $\mathrm{MKA}_{159}=5 \pm 1$ Gyr. In the sample, 134 of 159 systems are non magnetic (NMCV) having $\mathrm{MKA}_{\mathrm{NMCV}}=4.0 \pm 1.0 \mathrm{Gyr}$. In the sub-sample of NMCVs, 53 of 134 have $\mathrm{P}_{\text {orb }}<2.62 \mathrm{~h}$ and their MKA is $5.0 \pm 1.5 \mathrm{Gyr}$, whilst 81 of 134 systems have $\mathrm{P}_{\text {orb }}>2.62$ $\mathrm{h}$ and their MKA is $3.6 \pm 1.3 \mathrm{Gyr}$. This means that CVs below the 'period gap' are older than systems above the gap. This result in agreement with the standard evolution theory of CVs. The selection of $2.62 \mathrm{~h}$ as the border between the two groups of systems lies roughly in the middle of the 'period gap', where systems have been detected. This means that the 'period gap' does not exist anymore and the systems inside this 'gap' are just frontier objects between systems experiencing gravitational radiation and those experiencing magnetic braking. The reason because they are not so numerous as 

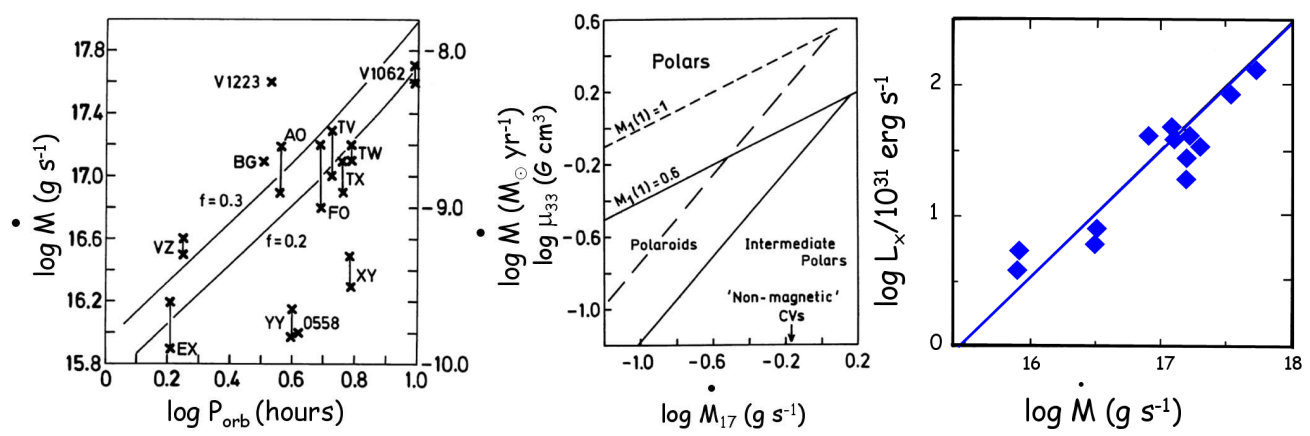

Figure 2: Left panel: Intermediate polars in the plane $\dot{\mathrm{M}}-\mathrm{P}_{\text {orb }}$. The two lines are lines of disc stability: stable above and dwarf nova outburst below. They were computed for $\mathrm{f}=0.2$ and 0.3 , with $R_{\text {out }}=\mathrm{f} \times \mathrm{a}$, being a the separation of the two stars in the system (by courtesy of Warner, 1996). Central panel: Magnetic moment in units of $10^{33} \mathrm{G} \mathrm{cm}^{3}$ versus mass accretion rate in units of $10^{17} \mathrm{~g} \mathrm{~s}^{-1}$. Boundaries for white dwarf mass $\mathrm{M}_{1}=1$ $\mathrm{M}_{\odot}$ and $\mathrm{M}_{1}=0.6 \mathrm{M}_{\odot}$ have been computed for $\mathrm{P}_{\text {orb }}=4 \mathrm{~h}$ (by courtesy of Warner, 1996). Right panel: Mass transfer rate onto white dwarf versus (2-10 keV) X-ray luminosity (by courtesy of Warner, 1996).

those placed at sides could be the relative shorter time of permanence in the 'gap', and then difficult to be detected.

Our opinion is that a more appropriate investigation of the class of the so-called IPCVs is necessary. Indeed, such systems could show surprises if deeply studied, as for instance occurred for SS Cyg. This system, usually considered a NMCV because of a classification once made by Bath \& van Paradijs (1983), whilst since 1984 it has been claimed as an IPCV by Giovannelli et al. (1985) and later confirmed several times (e.g., Giovannelli \& Martinez-Pais, 1991; Giovannelli, 1996; Giovannelli \& Sabau-Graziati, 1999, Gaudenzi et al., 2002). Giovannelli \& Sabau-Graziati (2012a) discussed all the circumstantial proofs in favor of the magnetic nature of SS Cyg, as well as those adverse, concluding with reasonable certainty that its nature is magnetic, being the magnetic field intensity $\mathrm{B}=1.7 \pm 0.8 \mathrm{MG}$, in agreement with the value $(\mathrm{B} \leq 1.9 \mathrm{MG})$ derived by Fabbiano et al. (1981) by using X-ray, UV and optical coordinated measurements.

This would teach a lesson: it is mandatory to observe CVs for long time in order to follow at least a whole period of the binary system between two successive outbursts. This is, of course, possible only for systems like dwarf novae where the almost periodical outbursts occur in time scales of weeks-months. Networks of robotic telescopes can help in this matter (Giovannelli \& Sabau-Graziati, 2012b)

However, we can say that CVs form a broad stellar family of highly variable and dynamical members. When it comes to explaining particulars about, e.g., the detailed interaction between the transferred matter and the WD's atmosphere; irregularities within regular photometric behaviour; turbulent transport in the disc; or the final fate of these objects, more is missing than what is known, rendering their study ever more challenging.
At least, CVs are natural multi-wavelength laboratories offering us the possibility of studying in detail the behaviour of plasma and radiation under extreme physical conditions. The understanding of stellar evolution, electromagnetism and polarization, mass and radiation transfer or 3-D geometrical effects, in a broad spectral range from hard X-rays to radio, is mandatory for improving the knowledge of the nature of CVs.

Variability, from milliseconds to hundreds of years, follows from different physical processes taking place in these systems and can be studied by means of several astronomical techniques. As our skills in developing further these techniques grow our understanding of the CVs insights also grows; and the more we learn about CVs the further techniques and theory develop. On the other hand, it is well known that conclusions obtained in the field of CVs have been extrapolated, upwards or downwards in scale, to other fields such as AGNs or LMXRBs, and vice versa. From such exchanges of information and results astrophysical research in general always benefits. Rapid oscillations in CVs are particularly interesting. As reviewed by Warner (2004), the rich phenomenology of dwarf nova oscillations (DNOs) and quasi-periodic oscillations (QPOs) observed in CVs favour the interpretation that these rapid brightness modulations (3 to 11,000 s timescales) are magnetic in nature - magnetically channelled accretion from the inner accretion disc for DNOs and possible magnetically excited traveling waves in the disc for QPOs. There is increasing evidence for the magnetic aspects, which extend to lower fields the well-known properties of strong field (PCVs) and intermediate strength field (IPCVs) CVs. The result is that almost all CVs show the presence of magnetic fields on their WD primaries, although for many the intrinsic field may be locally enhanced by the accretion process itself. There are many behaviour that parallel the QPOs seen in X-ray binaries, with 
high- and low-frequency X-ray QPOs resembling, respectively, the DNOs and QPOs in CVs. Other papers about rapid oscillations in CVs are those by Warner \& Woudt (2005) and Pretorius, Warner \& Woudt (2006).

The current estimate of the space density of CVs is of $\sim 3 \times 10^{-6} \mathrm{pc}^{-3}$ (Warner, 2001). This may be a significant underestimate of CVs space density, as discussed by Patterson (1984). Although densities from the most comprehensive optical Palomar-Green survey raises the estimate at $(3-6) \times 10^{-6} \mathrm{pc}^{-3}$, X-ray All-Sky surveys give densities of $\sim 1 \times 10^{-5} \mathrm{pc}^{-3}$ for detected systems of low $\dot{M}$ in hard X-rays (Patterson, 1998). Then from observational point of view, it is necessary an intensive search for the faint CVs predicted by population synthesis with orbital periods at $\sim 80-100$ min that have passed through the orbital period minimum at $\sim 78 \mathrm{~min}$ and have increasing orbital periods. This research must be done among the low $\dot{M}$ systems detected by X-ray surveys. Thanks to its high sensitivity, INTEGRAL is very useful for this purpose. Up to now, it discovered several new faint $\mathrm{CVs}$, with $\mathrm{P}_{\text {orb }}>3 \mathrm{hr}$, and only one with $\mathrm{P}_{\text {orb }}<3 \mathrm{hr}$ (e.g. Šimon et al., 2006; Hudec et al., 2008). High speed photometry of faint CVs have shown that: i) 1 of 10, TV Crv has $\mathrm{P}_{\text {orb }}=1.509 \mathrm{hr}$ (Woudt \& Warner, 2003); ii) 5 of 13 have $\mathrm{P}_{\text {orb }}<2 \mathrm{hr}$ (Woudt, Warner \& Pretorius, 2004); iii) 1 (CAL 86) of 12 has $\mathrm{P}_{\text {orb }}=1.587 \mathrm{hr}$ (Woudt, Warner \& Spark, 2005); iv) 3 of 11 have $\mathrm{P}_{\text {orb }}>3 \mathrm{hr}$ (Witham et al., 2007).

For reviews about CVs see the fundamental papers by Robinson (1976), Patterson $(1984,1994)$, Hack \& la Dous (1993), and the books of Warner (1995a) and Hellier (2001). More recent reviews are those by Connon Smith (2007), and Giovannelli (2008). The long review The Impact of Space Experiments on our Knowledge of the Physics of the Universe by Giovannelli \& SabauGraziati (2004) contains also a part devoted to CVs.

\section{Multifrequency Emissions}

In CVs there are several components that are responsible for the total emission. Deep discussions about these components can be found in the literature, but there is a recent review by Giovannelli (2008, and the references therein) that exhaustively summarize their contributions to the total multifrequency emission of CVs. Briefly, such components are:

a) The secondary stars are cool main sequence stars with spectral type ranging from G8 to M6, corresponding to temperatures from 5,000 to $3,000 \mathrm{~K}$. Their contribution is mainly in red and IR regions of the electromagnetic spectrum.

b) The primary stars. The temperatures of WDs are known only in few cases: when they belong to high inclination systems, or when they accrete matter with a very low mass transfer rate. However, the WD temperatures range between 10,000 and 50,000 K (Sion, 1986; 1991). Urban \& Sion (2006) found that the WDs in CVs above the period gap are hotter and more accretion heated $\left(\overline{\mathrm{T}_{\mathrm{eff}}}=25,793 \mathrm{~K}\right)$ than those below the gap $\left(\overline{T_{\text {eff }}}=18,368 \mathrm{~K}\right)$.

Therefore WDs are expected to radiate essentially in the UV, but they can be visible also in the optical range if they are not too hot.

c) The accretion disc: it does not have a homogeneous temperature, but spans a large range. Since the temperature distribution in discs is poorly known, in order to obtain a rough evaluation of their contribution to the total emission it is necessary to evaluate the contributions at different frequencies of a synthetic disc constituted of black bodies at different temperatures, the temperature distribution being that of a stationary accretion disc (e.g., la Dous, 1994). It then appears evident that the contribution of such an accretion disc is important in the whole range between EUV and IR, depending on the choice of the disc parameters. Furthermore, the UV radiation can be supplied from a zone in the vicinity of the WD (some ten stellar radii), which could contain any optically thick material left there. However, the argument of accretion discs deserves an important comment. It appeared evident that the viscosity of matter inside the accretion discs plays a fundamental role in the description of physical processes occurring there. In spite of numerous attempts in determining such a viscosity, the physical nature of that still remains largely indeterminate. The best training for study the viscosity is the subclass of dwarf novae, showing quasi-periodic outbursts which occur on a time scale from weeks to months (or even years) and are due to non-stationary accretion.

Meyer \& Meyer-Hofmeister (1981, 1982, 1983) firstly discussed the physical mechanism responsible for dwarf nova outbursts which is connected with the thermal instability of the disc which occurs in the temperature range corresponding to the ionization of hydrogen. Soon after Smak (1984a,b) extended the study of such a mechanism. The details are summarized by Smak (2002).

It is important to point out that the shapes of dwarf nova light curves, which depend on a number of relevant parameters, depend also on viscosity. In particular, the characteristic time-scales observed during outbursts depend on the viscous time-scale. This provides an important and almost unique opportunity of obtaining some constraints on viscosity or - within the $\alpha$ disc approach (Shakura \& Sunyaev, 1973) - of an empirical determination of $\alpha$.

For a more complete and detailed discussion of dwarf novae and models of their outbursts - see reviews by Cannizzo (1993), Osaki (1996), and Lasota (2001). 
The Golden Age of Cataclysmic Variables and Related Objects (Old and News)

d) The boundary layer: a very important zone for the emission is that of the transition between the accretion disc and the WD surface, namely the boundary layer. It is possible that all, or at least a significant fraction, of the kinetic energy of the material contained in the accretion disc must be radiated away within the geometrically very small boundary layer in order to have the possibility of the material accreting onto the WD's surface. Then, whatever the situation, one can assume the presence of a strong X-ray source at the boundary layer, which will be visible also in the EUV and shortwavelength UV according to the choice of disposable theoretical parameters.

Most of the radiation then comes from the accretion disc and boundary layer, which contribute roughly $50 \%$ each. From the accretion disc the radiation is essentially emitted in the optical and UV, whilst from the boundary layer - optically thick (which occurs at high accretion rates) - the radiation is emitted in the soft X-ray range; when the accretion is at low rates the boundary layer is optically thin and appears as a hard thermal bremsstrahlung source. These predictions have been tested experimentally, comparing the observations of CVs in optical, UV and soft X-ray ranges (e.g. Wood et al., 1989; Horne et al., 1994).

e) The gas stream: it is definitively optically thin and cool and contains rather little material; so, probably, its contribution to the total emission of CVs is negligible at all frequencies as source of continuum, whilst it could contribute to the formation of lines in the red and IR regions.

f) The hot line: the energy excess zone in the place where the stream comes to the disc is a shock wave. This zone was previously known as 'the hot spot'. Its structure and radiation characteristics are still an open problem; it is visible in many systems in optical photometry (less in the IR and never in the UV) as a periodically recurring hump in the orbital light curve. Its temperature must be $\leq 10,000 \mathrm{~K}$.

g) Hot corona or chromosphere: is a shell of optically thin and rather hot gas, below and above the accretion disc. X-ray and UV line radiation are tentatively attributed to it, whilst it probably does not contribute to the UV, optical or IR continuum emission.

Usually no radio emission from CVs has been measured. Only upper limits for individual systems of order of a few mJy are available (e.g $\leq 10 \mathrm{mJy}$ in SS Cyg Cordova, Mason \& Hjellming 1983). However, recently, Körding et al. (2008) detected a radio flare from SS Cyg peaked at $1.1 \mathrm{mJy}$ with a duration of order 20 days, above the upper limit of $0.08 \mathrm{mJy}$. This radio flare was simultaneous with the optical long outburst peaked at about $1 \mathrm{Jy}$.
Pringle \& Wade (1985) computed the contribution functions of the most important components of a cataclysmic system, previously discussed. The plot can be found also in Fig. 6 of Giovannelli's paper (2008).

During quiescence dwarf novae emit essentially hard $\mathrm{X}$-rays $(\sim 0.1-4.5 \mathrm{keV})$ and the flux distribution is rather well approximated by a thermal bremsstrahlung with $\mathrm{KT}_{\text {brems }} \approx 10 \mathrm{keV}$ (Cordova \& Mason, 1983). A direct correlation between the hard X-ray/optical fluxes ratio and $\mathrm{H}_{\beta}$ equivalent width has been found by Patterson \& Raymond (1985).

During outburst dwarf novae emit soft X-rays (0.18$0.5 \mathrm{keV}$ ) with an increase of the flux of the order of 100 or more, although most of the radiation is hidden in the EUV range (Cordova \& Mason, 1984). The soft X-ray spectra can be fitted either with black bodies at $\mathrm{KT}_{\mathrm{bb}} \approx$ 25-30 eV or, alternatively, with bremsstrahlung spectra at $\mathrm{KT}_{\text {brems }} \approx 30-40 \mathrm{eV}$.

The most important features are the anti-correlation between the hard and soft X-ray emissions during the outburst cycle and the correlation between soft X-ray and optical emissions, as measured for SS Cyg (Watson, King \& Heise, 1985), or - what is the same - anticorrelation between the hard X-ray and optical emission (Ricketts, King \& Raine, 1979). During an outburst of SS Cyg there is also the correlation of optical and EUV emissions, that are anti-correlated with the hard X-ray emission detected by RXTE (Wheatley et al., 2003).

What does that mean? The UV flux and the bulk of optical flux in dwarf novae and nova-like stars originate in the accretion disc. The IR flux observed during quiescence and possibly some of the optical flux come from the secondary late-type star. The rise to an outburst either occurs simultaneously at all wavelengths when it is slow, or progressively starts later with decreasing wavelengths when it is fast, since ever more central hotter parts of the disc become involved. Indeed, several dwarf novae have been observed in the UV and optical during the rise to maximum outburst brightness and their behaviour are quite similar: the UV rise lags the optical rise by up to a day (e.g., VW Hyi: Hassall et al. (1983); CN Ori and RX And: Cordova, Ladd \& Mason (1986); WX Hyi: Hassall, Pringle \& Verbunt (1985). With respect to the optical band, this lag is similar also in the EUV region covered by the Voyager (50-1200 $\AA$ ) for SS Cyg (Polidan \& Holberg, 1984) and VW Hyi (Polidan \& Holberg, 1987).

This fact strongly supports the origin of the outburst being in the cooler outer part of the disc rather than in the hotter parts near the WD; therefore mininova models for the outbursts are probably excluded (Cordova \& Howarth, 1987). The two models for triggering the outbursts, compatible with the lag observations, are then: 
- an instability in the secondary star which allows the transfer of more mass to the disc;

- a thermal instability in the outer disc, which results in material stored there being suddenly transported through the disc. During the decline, the whole disc cools simultaneously. The contribution to the total emission from the boundary layer between the disc and the WD surface is in UV and X-ray ranges: the boundary layer is optically thin during quiescence and then emits hard $\mathrm{X}$-rays, but it is optically thick during outburst and then emits soft $\mathrm{X}$-rays since the radiation is thermalized before escape (la Dous, 1993).

From the EUVE (Extreme Ultraviolet Explorer) Craig et al. (1997) have shown the EUV spectra of three NPCVs in outburst, namely VW Hyi, U Gem and SS Cyg. VW Hyi shows the softest EUV spectrum peaked at $\sim 250 \AA$. However, its boundary layer/disc luminosity ratio $-\mathrm{L}_{\mathrm{bl}} / \mathrm{L}_{\mathrm{disc}} \sim 0.2$ (Mauche et al., 1991) - is in contrast with the boundary layer models. In U Gem the total size of the EUV emitting region is comparable to that of the WD itself, which indicates that the outburst in mainly confined to the inner disc/boundary layer region (Long et al., 1996). Its $\mathrm{L}_{\mathrm{bl}} / \mathrm{L}_{\mathrm{disc}} \sim 1$. SS Cyg is the hardest of the three EUV NMCVs. Its EUV spectrum is rather complex and changed by a factor 100 during the outburst with an almost constant spectral energy distribution. Quasi-coherent oscillations $(\sim 7-9 \mathrm{~s})$ have been detected in the EUV emission (Mauche, 1996). SS Cyg shows no EUV emission longward $\sim 130 \AA$ A. Mauche, Raymond \& Mattei (1995) found that for SS Cyg the relation $\mathrm{L}_{\mathrm{bl}} / \mathrm{L}_{\mathrm{disc}} \sim 1$, valid for the boundary layer models is strongly violated, being this ratio $\mathrm{L}_{\mathrm{bl}} / \mathrm{L}_{\mathrm{disc}} \sim 0.07$. And this is one more proof that SS Cyg is not a NMCV — as already remarked - as claimed by Giovannelli's group.

IUE satellite deserves special comments since it was fundamental in improving the knowledge of CVs. A detailed review can be found in Giovannelli (2008). Briefly, the IUE gave significant contributions on:

i) Knowledge of disc accreting and magnetic CVs, as extensively discussed by Cordova (1995), and references therein.

ii) Nature of the high velocity winds. During outburst of SS Cyg the spectral emission features disappear or go into absorption, some of them showing P Cygni profile (e.g. CIV), which clearly indicate the presence of high velocity wind from the system. The emission features appear again when the system is going into quiescence (Giovannelli et al., 1990).

iii) Boundary layer emission.

Multifrequency observations show that X-ray luminosity at all outburst phases is much lower (about at least a factor 10) than the UV/optical luminosity from the disc, as expected from the models (e.g., Mauche, 1998). This simply means that the boundary layer models are not correct.

iv) Underlying WD and its photosphere. IUE provided the first evidence that the WD is heated by the dwarf nova outburst and subsequently cooled. A list of nine such systems has been reported by Szkody (1998). These measurements are very difficult because of the long quiescence-outburst-quiescence cycles (from weeks to years). The short outburst period dwarf nova VW Hyi cooled to $18,000 \mathrm{~K}$ (from 20,500 K) in the 14 days before the next outburst began (Verbunt et al., 1987).

v) Magnetic field of the WD. Indirect evaluations of magnetic field intensities in CV WDs have been obtained through multifrequency observations (e.g., Fabbiano et al., 1981). Our feeling is that the problem of magnetic fields in WDs has been underestimated in the studies of CVs.

Too many simplified models of disc accreting and magnetic CVs have been developed under the hypothesis that CVs can be sharply divided into three classes: Polar, IP, Non-Magnetic. Magnetic fields are smoothly varying in their intensities from one class to another. The discovery in some IPs of a circularly polarized optical emission suggests that these intermediate polars will evolve into polar systems (e.g., Mouchet, BonnetBidaud \& de Martino et al., 1998). Some evidence of the continuity between the IPCVs and PCVs is coming from the detection of the SW Sex systems. They have orbital periods just inside the so-called 'period gap', which separates the two classes of IPCVs and PCVs (e.g. Rodriguez-Gil, 2003 and references therein; Rodriguez-Gil et al., 2007).

Looking at the homogeneous set of data coming from the IUE for PCVs and IPCVs, it has been possible to obtain important information on common properties and peculiarities of these binaries (de Martino, 1999 and references therein), which render the two classes rather similar in some of their UV behaviour. Mouchet, Bonnet-Bidaud \& de Martino (1998), and de Martino (1998) made the hypothesis that the two classes are evolutionary related.

The far UV vs near UV colour-colour diagram for MCVs was constructed by de Martino (1999). Such a diagram was constructed measuring broad band continua in the IUE short wavelength range (1420-1520 $\AA$ and $1730-1830 \AA$ ) and in the long wavelength range (2500-2600 $\AA$ and 2850-2900 $\AA$ ). Clearly the UV continua cannot be simply described by a single component but possess different contributions, as discussed by de Martino (1999) and already noted in the past, since 1984, by Giovannelli et al. (1985).

Araujo-Betancor et al. (2005) obtained Hubble Space Telescope (HST) STIS data for a total of 11 PCVs 
The Golden Age of Cataclysmic Variables and Related Objects (Old and News)

as part of a program aimed at compiling a homogeneous database of high-quality FUV spectra for a large number of CVs. Comparing the WD temperatures of PCVs with those of NMCVs, they find that at any given orbital period the WDs in PCVs are colder than those in NMCVs. The temperatures of WDs in PCVs below the period gap are consistent with gravitational radiation as the only active angular momentum loss mechanism. The differences in WD effective temperatures between $\mathrm{PCV}$ s and NMCVs are significantly larger above the period gap, suggesting that magnetic braking in PCVs might be reduced by the strong field of the primary. Araujo-Betancor et al. (2005) derive a lower limit on the space density of PCVs of $1.3 \times 10^{-6} \mathrm{pc}^{-3}$.

\section{Renewed Interest for Cataclysmic Variables}

Before the advent of ROSAT X-ray satellite, MCVs were relegated to a subsection of conferences about CVs that were mainly concentrated on NMCVs. The ROSAT satellite discovered many MCVs that even menaced to overthrow our understanding of the secular evolution of 'normal' CVs by appearing - apparently inexplicable - in the so-called 'period gap' in the orbital-distribution of CVs (e.g. Vrielmann \& Cropper, 2004). But in spite of this, CVs were not considered, in general, for many years as principal targets of high energy X-ray experiments.

At the beginning of the nineties of the last century, acceleration of particles by the rotating magnetic field of the WD in intermediate polars in the propeller regime - AE Aqr -- detected by ground-based Cherenkov telescopes in the TeV passband (e.g. Meintjes et al. 1992), and $\mathrm{TeV}$ emission from the polar AM Her detected by ground-based Cherenkov telescopes (Bhat et al. 1991) - measurements never confirmed - were the main reasons of renewed interest for CVs in the high energy astrophysicists community.

The INTEGRAL observatory, until the beginning of 2007, had observed over 70 percent of the sky, with a total exposure time of 40 million seconds. Bird et al. (2007) published the third INTEGRAL catalogue of gamma-ray sources. It contains a total of 421 gammaray objects. Most have been identified as either binary stars in our Galaxy containing exotic objects such as black holes and neutron stars, or active galaxies, far away in space. But a puzzling quarter of sources remain unidentified so far. They could be either star systems enshrouded in dust and gas, or CVs. Integral observes in the gamma-ray band so it can see through the intervening material. It has demonstrated that it can discover sources obscured at other wavelengths. One surprise has been the efficiency with which Integral has detected just one minor subclass CVs, the so- called IPCVs. Initially astronomers were not sure that CVs would emit gamma rays. Indeed, INTEGRAL has already shown that only about one percent of them do. This fact overbearingly renewed the interest for $\mathrm{CVs}$, apparently fallen into disgrace in favour of binary systems containing either neutron stars or black holes. The fourth IBIS/ISGRI catalog reports 331 additional sources when compared to the third catalog. Of these, 120 are associated with extragalactic sources, while only 25 are associated with known Galactic sources, and the remainder are so far unidentied (Bird et al. 2010). CVs constitute $\sim 5 \%$ of the total sources.

Moreover, since the CVs measured by the INTEGRAL observatory are magnetic in nature, the interest for such class of objects has been addressed to evolutionary problems.

The long-standing fundamental predictions of evolution theory are finally being tested observationally. All facets of the accretion process in CVs, including variability, disc winds and jets, are universal with accreting WDs, neutron stars, and black holes (Knigge, 2010, 2011). Knigge, Baraffe \& Patterson (2011) extensively discussed the reconstruction of the complete evolutionary path followed by CVs, based on the observed mass-radius relationship of their donor stars, following Knigge (2006) that discussed the observational and theoretical constraints on the global properties of secondary stars in CVs using the semi-empirical CV donor sequence, and concluded that most CVs follow a unique evolutionary track.

In the standard model of CV evolution, angularmomentum-loss (AML) below the period gap are assumed to be driven solely by gravitational radiation (GR), while AMLs above the gap are usually described by a magnetic bracking (MB) (Rappaport, Verbunt \& Joss (1983). Knigge, Baraffe \& Patterson (2011) with their revised model, found the optimal scale factors $\mathrm{f}_{\mathrm{GR}}$ $=2.47$ below the gap and $\mathrm{f}_{\mathrm{MB}}=0.66$ above, whilst the standard model gives $\mathrm{f}_{\mathrm{GR}}=\mathrm{f}_{\mathrm{MB}}=1$. This revised model describes the mass-radius data much better than the standard model.

The sub-class of CVs, named Classical Novae $(\mathrm{CNe})$, which are the third more powerful stellar explosions in a galaxy, have been observed as close as a kpc and as far as galaxies in Fornax cluster. The time to report on the recent renaissance in studies on $\mathrm{CNe}$ thanks to observations with $8-10 \mathrm{~m}$ class telescopes, high resolution spectroscopy, in synergy with observations from space carried out with Swift, XMM, Chandra, HST, and Spitzer, coupled with recent advances in the theory of the outburst, seems now in order. Moreover, the possible connection among some CV-types and SNe-Ia will definitively justify the renewed interest about CVs. 


\section{Classical and Recurrent Novae}

Classical novae are expected to recur on timescales from 100,000 years to just a few decades. The most important physical parameters controlling this recurrence timescale are the WD mass, and the mass accretion rate from the secondary (e.g. Yaron et al. 2005). Once classical nova $(\mathrm{CN})$ is recorded more than once, it can be designated as "recurrent" (RN). Since the WD and the binary system remain intact after an outburst, it is possible that classical novae may actually be the same as recurrent novae if observed over a long enough time period. While the interval between outbursts of recurrent novae range from 10 to 100 years, it has been estimated that the time interval for classical novae would range from about 30,000 years for a $1.3 \mathrm{M}_{\odot}$ WD to 100,000 years for a $0.6 \mathrm{M}_{\odot}$ WD. Given long enough - it is expected that all classical novae will be observed as recurrent novae.

The long term behaviour of classical old novae, and the optical behaviour of CNe in outburst were discussed by Bianchini (1990), and Seitter (1990), respectively. The books by Cassatella \& Viotti (1990) and by Bode $\&$ Evans (2008) are very useful for studying the physics of classical novae.

Recurrent novae are a rare sub-class of cataclysmic variable stars; WDs accreting material from a binary companion in which more than one classical nova-type outburst has been observed (see the book of Hellier, 2001 for a comprehensive review of CVs). Nova outbursts are suspected to be due to a thermonuclear runaway on the surface of the WD, which releases huge amounts of thermal energy once a critical pressure is reached at the base of the shell of accreted material.

One of the most interesting $\mathrm{RNe}$ is RS Ophiuchi (RS Oph). It is an amazingly prolific recurrent nova, with recorded outbursts in 1898, 1907, 1933, 1945, 1958, 1967, 1985 and 2006 (Schaefer 2010). The short time between outbursts $(\sim 20$ yrs $)$ suggests that RS Oph hosts a massive WD accreting material at a significantly high rate.

In the latter paper Schaefer discussed not only RS Oph, but also the photometric histories of all known galactic RNe.

Classical and recurrent nova outbursts have been recently discussed by Bode (2011a,b) and Evans (2011). The proceedings of a conference about RS Oph and recurrent phenomenon can be very useful for details (Evans et al., 2008). General properties of quiescent novae have been discussed by Warner (2002). The very useful book of Bode \& Evans (2008) about classical novae examines thermonuclear processes, the evolution of nova systems, nova atmospheres and winds, the evolution of dust and molecules in novae, nova remnants, and observations of novae in other galaxies. It includes observations across the electromagnetic spectrum, from radio to gamma rays, and discusses some of the most important outstanding problems in classical nova research.

Of the $\sim 400$ known Galactic classical novae, only 10 of them are recurrent. Eight of them harbour evolved secondary stars, contrary to classical novae that contain main sequence stars (Darnley et al., 2011). They propose a new nova classification based on the evolutionary state of the secondary star, contrary the current schemes based on the properties of outbursts. Such classification contains three groups of novae: i) Main Sequence Nova (MS-Nova); ii) Sub-Giant Nova (SGNova); and iii) Red Giant branch Nova (RG-Nova).

RNe play an important role in the studies of SN Ia progenitors (Surina et al., 2011). RNe are likely progenitors of Type-Ia supernovae.

In order to brave this important problem the use of archival data is the only way to answer the big question. Now, huge and comprehensive set of archival RN data go back to 1890 .

\section{Progenitors of SN Ia}

It is well accepted by the community that Type-Ia SNe are the result of the explosion of a carbon-oxygen WD that grows to near Chandrasekhar's limit in a close binary system (Hoyle \& Fowler, 1960). But the debate is focussed around the different kinds of progenitors. Indeed, in the past, two families of progenitor models have been proposed. They differ in the mode of WD mass increase. The first family is the so-called single degenerate (SD) model (Whelan \& Iben, 1973), in which the WD accretes and burns hydrogen-rich material from the companion. The second family is the so-called double degenerate (DD) model, in which the merging of two WDs in a close binary triggers the explosion (Webbing, 1984; Iben \& Tutukov, 1984). The two scenarios produce different delay times for the birth of the binary system to explosion. Thus it is hopefully possible to discover the progenitors of Type-Ia SNe by studying their delay time distribution (DDT). The DDT can be determined empirically from the lag between the cosmic star formation rate and Type-Ia SN birthrate.

The energy released through runaway thermonuclear process ejects the majority of the unburnt hydrogen from the surface of the star in a shell of material moving at speeds of up to $1.5 \times 10^{3} \mathrm{~km} \mathrm{~s}^{-1}$. This produces a bright but short-lived burst of light - the nova.

Although Type-Ia supernova appear to have similar origin to classical novae, there are key differences. The most important is that in a classical nova, the thermonuclear runaway occurs only on the surface of the star, allowing the WD and the binary system to remain intact (e.g. Townsley \& Bildsten, 2005). In a Type-Ia 
The Golden Age of Cataclysmic Variables and Related Objects (Old and News)

supernova, the thermonuclear runaway occurs within WD itself, completely disrupting the progenitor. This is reflected in the amount of energy released in the explosions, with classical novae releasing $\sim 10^{44} \mathrm{erg}$, and Type-Ia supernovae $\sim 10^{51} \mathrm{erg}$.

The possible progenitors of SN Ia are: i) Recurrent Novae; ii) Symbiotic stars; iii) Super-soft sources; iv) Double WD Binaries; and v) WDs accreting material from red-giant companions.

i) Recurrent Novae are just a subset of ordinary novae that happen to go off more than once per century.

As such, they are binary systems with matter flowing off a companion star onto a WD, accumulating on its surface until the pressure gets high enough to trigger a thermonuclear runaway that is the nova.

Only $10 \mathrm{RNe}$ are known in our Milky Way galaxy, including: U Sco (1863, 1907, 1917, 1936, 1945, 1969, 1979, 1987, 1999); Т Pyx (1890, 1902, 1920, 1944, 1967); T CrB (1866, 1946); RS Oph $(1898,1907,1933$, 1945, 1958, 1967, 1985, 2006).

To recur with $\tau_{\text {rec }}<100$ years, RNe must have: high WD mass $\left(1.2 \mathrm{M}_{\odot}<\mathrm{M}_{\mathrm{WD}}<\mathrm{M}_{\text {Chandra }}\right)$, and high accretion rate $\left(\dot{\mathrm{M}} \sim 10^{-7} \mathrm{M}_{\odot} \mathrm{yr}-1\right)$. SN Ia occurs if: i) the mass ejected for each eruption is less than the mass accreted onto the WD $\left(\mathrm{M}_{\text {ejected }}<\dot{\mathrm{M}} \tau_{\text {rec }}\right)$; ii $)$ the rate of death $\mathrm{RNe}$ must be enough to produce the SN Ia rate $\left(R_{R N d e a t h}=R_{\text {SNIa }}\right)$, being $R_{R N d e a t h}=N_{R N}$ $\times\left(0.2 \mathrm{M}_{\odot} \dot{\mathrm{M}}\right)$.

In order to solve the problems we need to know $\tau_{\text {rec }}$ (recurrence time scale) from archive plates, $\mathrm{N}_{\mathrm{RN}}$ (number of RNe in the Milky Way) from archive plates and AAVSO, $\dot{M}$ (mass accretion rate onto WD) from the average in the last century, $\mathrm{M}_{\text {ejected }}$ (mass ejected in eruption) from pre-eruption eclipse timing.

Some results have been obtained for becoming optimists in solving the problem of SN Ia production. Indeed Schaefer (2011) obtained for CI Aql and U Sco $\left.\mathrm{M}_{\text {ejected }}<<\dot{\mathrm{M}} \tau_{\text {rec }}\right)$.

Thus, WDs are gaining mass and the latter RNe will collapse as SN Ia. Moreover, for the Milky Way, M31, and LMC $\mathrm{R}_{\mathrm{RNdeath}} \sim \mathrm{N}_{\mathrm{RN}}$. Then there are enough RNe to supply the Type-Ia SN events.

ii) Symbiotic Stars contain WDs efficiently accreting material from the secondary star. In most cases they steadily burn $\mathrm{H}$-rich material allowing them to grow in mass. Some of these systems can produce high mass WDs. In symbiotic RNe (SyRNe) the WD mass is already very close to Chandrasekhar's limit. For instance in V 407 Cyg a very massive WD is accreting material at a rate of $\sim 10^{-7} \mathrm{M}_{\odot} \mathrm{yr}^{-1}$ from a Miratype companion (Mikołajewska, 2011).

iii) Super-soft Sources are probably WDs that accrete material and burn hydrogen. Voss \& Nielemans (2008) discovered an object at the position of the TypeIa SN2007on in the elliptical galaxy NGC1404 on pre- supernova archival X-ray images. This result favours the accretion model (SD) for this supernova, although the host galaxy is older than the age at which the explosions are predicted in SD models. However, the DD model cannot be ruled out by this event because a hot accretion disc is probably the intermediate configuration of the system, between first WD-WD Roche-lobe contact and explosion (Yoon, Podsiadlowski \& Rosswog, 2007).

Greggio, Renzini \& Daddi (2008) starting from the fact that Type-Ia SN events occur over an extended period of time, following a distribution of delay times (DDT), discussed theoretical DDT functions that accommodate both "prompt' and 'tardy" SN events derived by empirically-based DDT functions. Moreover such theoretical DDT functions can account for all available observational constraints. The result is that $\mathrm{SD} / \mathrm{DD}$ mix of SNIa's is predicted to vary in a systematic fashion as function of cosmic time (redshift).

iv) Double WDs Binaries are systems containing two WDs that can merge and giving rise to SN explosion. Yoon, Podsiadlowski \& Rosswog (2007) explored the evolution of the merger of two carbon-oxygen (CO) WDs. Their results imply that at least some products of double CO WDs merger may be considered good candidates for the progenitors of Type-Ia SNe. Brown et al. (2011) and Kilic et al. (2011) studied a complete colour-selected sample of double-degenerate binary systems containing extremely low mass (ELM) $\left(\leq 0.25 \mathrm{M}_{\odot}\right)$ WDs. Milky Way disc ELM WDs have a merger rate of $\approx 4 \times 10^{-5} \mathrm{yr}^{-1}$ due to gravitational wave radiation. The ELM WD systems that undergo stable mass transfer can account for about $3 \%$ of AM CVn stars. The most important fact is that the ELM WD systems that may detonate merge at a rate comparable to the estimate rate of underluminous SNe. These SNe are rare explosions estimated to produce only $\sim 0.2$ $\mathrm{M}_{\odot}$ worth of ejecta. At least $25 \%$ of ELM WD sample belong to the old tick disc and halo components of our Galaxy. Thus, if merging ELM WD systems are the progenitors of underluminous $\mathrm{SNe}$, transient surveys must find them in both elliptical and spiral galaxies.

v) WDs accreting material from red-giant companions. Observations carried out by Patat et al. (2008) with VLT-UVES allowed to detect circumstellar material in a normal Type-Ia SN. The expansion velocities, densities and dimensions of the circumstellar envelope indicate that this material was ejected from the system prior to the explosion. The relatively low expansion velocities favour a progenitor system where a WD accretes material from a companion star, which is in the red-giant phase at the time of explosion.

Bianco et al. (2011) searched for a signature of a non-degenerate companion in three years of Supernova 
Legacy Survey data. They found that a contribution from WD/red-giant binary system to Type-Ia SN explosions greater than $10 \%$ at $2 \sigma$, and than $20 \%$ at $3 \sigma$ level is ruled out.

Type-Ia SNe are used as primary distance indicators in cosmology (e.g. Phillips, 2005). Phillips (2011) reviewed the near-infrared (NIR) of Type-Ia SNe concluding that such SNe are essentially perfect standard candles in the NIR, displaying only a slight dependence of peak luminosity on decline rate and colour. Lira (1995) first noted that B-V evolution during the period from 30 to 90 days after $\mathrm{V}$ maximum is remarkably similar for all SN Ia events, regardless of light-curve shape. This fact was used by Phillips et al. (1999) to calibrate the dependence of the $B_{\max }-V_{\max }$ and $V_{\max }{ }^{-}$ $\mathrm{I}_{\max }$ colours on the light curve parameter $\Delta \mathrm{m}_{15}$ (B) which can, in turn, be used to separately evaluate the host galaxy extinction. Using these methods for eliminating the effect of the reddening, they reanalyzed the functional form of the decline rate versus luminosity relationship and gave a value of the Hubble constant of $\mathrm{H}_{0}=63.3 \pm 2.2 \pm 3.3 \mathrm{~km} \mathrm{~s}^{-1} \mathrm{Mpc}^{-1}$.

The use of Type-Ia SNe is also fundamental for determining some cosmological constraints, such as $\Omega_{\mathrm{M}}$ and $\Omega_{\Lambda}$ that fit a $\Lambda \mathrm{CDM}$ models with values of $0.211 \pm 0.034$ (stat) \pm 0.069 (sys) using a set of 252 highredshift SNe (Guy et al., 2010) and $0.713_{-0.029}^{+0.027}$ (stat) ${ }_{-0.039}^{+0.036}$ (sys) using a set of low-redshift nearby-Hubbleflow SNe (Kowalski et al., 2008), respectively.

In order to explore the difficult topic of the expansion of the Universe it is necessary to know the evolution of metallicity in old Universe that changes the Hubble Diagram shape. The proposed space observatory Super Nova Acceleration Probe (SNAP) is designed to measure the expansion of the Universe and to determine the nature of the mysterious Dark Energy that is accelerating this expansion. SNAP is being proposed as part of the Joint Dark Energy Mission (JDEM) (Stril et al., 2010), which is a cooperative venture between NASA and the U.S. Department of Energy. If selected it will be launched before 2020 .

SNAP cannot achieve its main goal without progenitor/evolution solution.

For comments and prospects about Type-Ia SN science in the decade 2010-2020 see the paper by Howell et al. (2009).

\section{Some Open Questions}

Several fundamental questions concerning CVs still remain waiting for a proper answer. We will present briefly only some of them here.

One of them is the lack of a coherent classification, especially for NLs. On the other hand, in gross features and in most respects, DN and NLs, as well as quiescent novae, are almost indistinguishable, although, in addition to their different outbursts' behaviour, there appear to be some further minor differences which are not yet understood (see Hack \& la Dous 1993). The question arises of whether the outburst behaviour, the current basis of almost all classification is really a suitable criterion for sorting CVs in physically related groups. There are also too many exceptions, either systems that do not fit in any particular group or that can be included in several of them, to be able to render the observational behaviour, at least as it is used at the present, suitable.

Could CVs be considered simply gravimagnetic rotators? This should be the most suitable approach for studying them from a physical point of view.

Studies of rotational equilibria of MCVs predict that IPCVs will evolve either into PCVs or into low field strength polars - presumably unobservable, and possibly EUV emitters - depending on their magnetic moments and orbital periods. Indeed, there are systems, like EX Hya-type, having magnetic moment similar to IPCVs above the 'period gap' and comparable to the weakest field AM Her-like systems.

Moreover, the detection of several SW Sex systems having orbital periods inside the so-called 'period gap' opens a new interesting problem about the continuity in the evolution of CVs.

The rare AM Canum Venaticorum (AM CVn) stars have extremely short orbital periods, between 10 and 65 minutes, and their spectra show no evidence for hydrogen. They appear to be helium-rich versions of CVs. They are still waiting for a general model. They are probably binary systems of two white dwarfs, but even this is still controversial.

Despite all the work developed during the last decades, the problem of modeling accretion discs in CVs is by no means closed, especially in quiescence. Closely related is the problem of the cause of outbursts. We really do not know which of the present two families of models (Disc Instability Models or Secondary Instability Models) is responsible for the CVs outburst phenomenon, or in which system is each model valid, although Martinez-Pais et al. (1996) gave a contribution in solving this problem at least in the case of SS Cygni; they found some evidence for an increase of the mass transfer rate from the secondary star as the mechanism responsible for symmetric outbursts. Something similar can be said about the super-outburst phenomenon in SU UMa systems.

Gaudenzi et al. (1990), analyzing IUE spectra of SS Cygni, discussed about the outburst production as due to the destruction of the accretion disc. The matter, passing through the boundary layer, slowly accretes onto the WD. Long and short outbursts correspond to 
The Golden Age of Cataclysmic Variables and Related Objects (Old and News)

total or partial destruction of the disc, respectively.

Alternatively, could nuclear burning be responsible of the production of outbursts in CVs? Indeed, nuclear burning onto white dwarf' surface was proposed by Mitrofanov $(1978,1980)$ as a mechanism suitable to generate X-rays in CVs. In spite of this shrewd suggestion, the community of theoreticians did not consider such a mechanism - certainly possible - worthy of taking up a part of their time. However, we believe that this alternative solution in explaining the generation of outbursts in CVs would deserve theoretician community's care. For instance, the white dwarf surface interested in the accretion in the system SS Cygni has been evaluated as $24 \%$ of the total (Gaudenzi et al., 2002). There, nuclear burning could occur.

Accretional heating by periodic DN events increases substantially the surface temperature of the WD in CVs (Godon \& Sion, 2002). Then, the envelope thermal structure resulting from compression and irradiation should be a crucial component in understanding the envelope structure of a pre-nova WD.

Another problem still open is connected with the classification of CVs in three kinds, namely NMCVs, PCVs and IPCVs. This is, in our opinion, another convenient classification, although artificial, probably not necessary if CVs are studied as gravimagnetic rotators. In this way a smooth evolution of the systems could be responsible of the variations of the gravimagnetic parameters.

Are the IPCVs and PCVs smoothly connected via the SW Sex-like systems placed just in between? SW Sex systems have indeed orbital periods belong to the so-called 'period gap', and then their presence there sure cancel that gap.

Could some systems behave in different ways depending on their instantaneous physical conditions? For this reason they could apparently behave sometimes as PCVs and sometimes as NPCVs.

An example very clear is that of SS Cygni, usually classified as a non-magnetic dwarf nova. It has been detected by the INTEGRAL observatory in a region of the spectrum (up to $\sim 100 \mathrm{keV}$ ). This emission is very hard to be explained without the presence of polar caps in the WD of the system. Several proofs have been shown and discussed many times by Giovannelli's group in order to demonstrate the Intermediate Polar nature of it (e.g., Giovannelli, 1996, and references therein; Giovannelli \& Sabau-Graziati, 1998; 2012a); indeed, SS Cygni shows characteristics of a NMCV, as well as those of IP and sometimes even those of polars, although its position in the $\log \mathrm{P}_{\text {spin }}-\log \mathrm{P}_{\text {orb }}$ plane is very close to the line where IPs lie.

Important results are coming from the SPITZER space telescope with the detection of an excess (3-8) $\mu \mathrm{m}$ emission from Magnetic CVs, due to dust (How- ell et al., 2006; Brinkworth et al., 2007). Gaudenzi et al. (2011) discussed about the reasons of the variable reddening in SS Cyg and demonstrated that this reddening is formed by two components: the first is interstellar in origin, and the second (intrinsic to the system itself) is variable and changes during the evolution of a quiescent phase. Moreover, an orbital modulation also exists. The physical and chemical parameters of the system are consistent with the possibility of formation of fullerenes.

The SPITZER space telescope detected the presence of fullerenes in a young planetary nebula (Cami et al., 2010). Fullerenes are the first bricks for the emergence of the life. Therefore, the possible presence of fullerenes in CVs opens a new line of investigation, foreboding of new interesting surprises.

\section{Conclusions}

At the end of this review it appears evident that the most suitable approach for studying CVs from a physical point of view is to consider them as gravimagnetic rotators.

The detection of several SW Sex systems having orbital periods inside the so-called 'period gap' opens a new interesting problem about the continuity in the evolution of CVs. Are the IPCVs and PCVs smoothly connected via the SW Sex-like systems placed just in between?

In order to fully understand the emission properties and evolution of $\mathrm{CVs}$, the mass-transfer process needs to be clearly understood, especially magnetic mass transfer, as well as the properties of magnetic viscosity in the accretion discs around compact objects. Consequently, the investigation on the magnetic field intensities in WDs appears crucial in understanding the evolution of CVs systems, by which it is possible to generate classical novae (e.g., Isern et al., 1997) and type-Ia supernovae (e.g., Isern et al., 1993).

In those catastrophic processes the production of light and heavy elements, and then the knowledge of their abundances provides strong direct inputs for cosmological models and cosmic ray generation problems.

Acknowledgments This research has made use of NASA's Astrophysics Data System.

\section{References}

[1] Andronov, I.L., Chinarova, L.L., Han, W., Kim, Y., Yoon, J.-N.: 2008, A\&A, 486, 855.

[2] Ak, T., Bilir, S., Ak, S., Coşkunoğlu, K.B., Eker, Z.: 2010, New Astron. 15, 491. doi:10.1016/j.newast.2009.11.007 
[3] Araujo-Betancor, S., Gänsicke, B.T., Long, K.S.,; Beuermann, K., de Martino, D., et al.: 2005, ApJ, 622, 589.

[4] Bath, G.T., van Paradijs, J.: 1983, Nature, 305, 33. doi:10.1038/305033a0

[5] Bhat, C.L., Kaul, R.K., Rawat, H.S., Senecha, V.K., Rannot, R.C., et al.: 1991, ApJ, 369, 475 doi:10.1086/169776

[6] Beuermann, K.: 1998, in High Energy Astronomy and Astrophysics, P.C. Agrawal \& P.R.

Visvanathan (eds.), India Univ. Press, p. 100.

[7] Bianchini, A.: 1990, in Physics of Classical Novae, A. Cassatella \& R. Viotti (eds.), Springer-Verlag, Lecture Notes in Physics, 369, 13.

[8] Bianco, F.B., Howell, D.A., Sullivan, M., Conley, A., Kasen, D., et al.: 2011, ApJ, 741, 20. doi:10.1088/0004-637X/741/1/20

[9] Bird, A.J., Malizia, A., Bazzano, A., Barlow, E.J., Bassani, L., et al.: 2007, ApJS, 170, 175. doi:10.1086/513148

[10] Bird, A.J., Bazzano, A., Bassani, L., Capitanio, F., Fiocchi, M., et al.: 2010, ApJS, 186, 1. doi:10.1088/0067-0049/186/1/1

[11] Bode, M.F.: 2011a, arXiv: 0911.5254v3.

[12] Bode, M.F.: 2011b, arXiv: 111.4941v1.

[13] Bode, M.F., Evans, A. (eds.): 2008, Classical Novae, Cambridge Astrophys. Ser., No. 43, Cambridge University Press.

[14] Brinkworth, C.S., Hoard, D.W., Wachter, S., Howell, S.B., Ciardi, D.R., et al: 2007, in 15th European Workshop on WDs, R. Napiwotzki \& M. R. Burleigh (eds.), ASP Conf. Ser. 372, 333.

[15] Brown, W.R., Kilic, M., Allende Prieto, C.A., Kenyon, S.J.: 2011, MNRAS, 411, L31. doi:10.1111/j.1745-3933.2010.00986.x

[16] Cannizzo, J.K., 1993, ApJ, 419, 318. doi:10.1086/173486

[17] Cassatella, A., Viotti, R. (eds.): 1990, Physics of Classical Novae, Springer-Verlag, Lecture Notes in Physics, 369. doi:10.1007/3-540-53500-4

[18] Charles, P.A., Seward, F.D., 1995, in Exploring the Universe, Cambridge University Press, UK, p. 21.

[19] Connon Smith, R.: 2007, astro-ph/0701654.
[20] Cordova, F.A.: 1995, in X-Ray Binaries, W. Lewin, J. van Paradijis \& E. van den Heuvel (eds.), Cambridge University Press, p. 331.

[21] Cordova, F.A., Mason, K.O.: 1983, in Accretion-Driven Stellar X-Ray Sources, Cambridge University Press, p. 154.

[22] Cordova, F.A., Mason, K.O., Hjellming, R.M.: 1983, PASP, 95, 69

[23] Cordova, F.A., Mason, K.O.: 1984, MNRAS, 206, 879 .

[24] Cordova, F.A., Ladd, E.F., Mason, K.O.: 1986, in Magnetospheric Phenomena in Astrophysics, R.I. Epstein \& W.C. Feldman (eds.), AIP, 144, 250.

[25] Cordova, F.A., Howarth, I.D.: 1987, in Exploring the Universe with the IUE Satellite, Y. Kondo (ed.), D. Reidel Publ. Co., Dordrecht, Holland, p. 395.

[26] Craig, N., Abbott, M., Finley, D., Jessop, H., Howell, S.B.,et al.: 1997, ApJS 113, 131.

[27] Darnley, M.J., Ribeiro, V.A.R.M., Bode, M.F., Hounsell, R.A., Williams, R.P.: 2011, arXiv: $1112.2589 \mathrm{v} 1$.

[28] Davis, P.J., Kolb, U., Willems, B., Gänsicke, B.T.: 2008, MNRAS, 389,1563.

[29] la Dous, C.: 1989, A\&A, 211, 131.

[30] la Dous, C.: 1993, in Cataclysmic Variables and Related Objects, M. Hack \& C. la Dous (eds.), NASA SP-507, 15

[31] la Dous, C.: 1994, SSR, 67, 1.

[32] Evans, A., Bode, M.F., O'Brien, T.J., Darnley, M.J. (eds.): 2008, RS Ophiuchi (2006) and the Recurrent Nova Phenomenon, ASP Conf. Ser., Vol. 401.

[33] Evans, A.: 2011, in Asymmetric Planetary Nebulae 5 Conference, A.A. Zijlstra, F. Lykou, I. McDonald, \& E. Lagadec (eds.), Jodrell Bank Centre for Astrophysics, p. 305.

[34] Fabbiano, G., Hartmann, L., Raymond, J. Steiner, J., Branduardi-Raymond, G., Matilsky, T.: 1981, ApJ, 243, 911.

[35] Forman, W., Jones, C., Cominsky, L., Julien, P., Murray, S., et al.: 1978, ApJS, 38, 357.

[36] Gaudenzi, S., Giovannelli, F., Lombardi, R., Claudi, R.: 1990, AcA, 40, 105 
The Golden Age of Cataclysmic Variables and Related Objects (Old and News)

[37] Gaudenzi, S., Claudi, R.U., Giovannelli, F., Lombardi, R., Pelosi, M., Strappolini, M.: 2002, in Multifrequency Behaviour of High Energy Cosmic Sources, F. Giovannelli \& L.

Sabau-Graziati (eds.), Mem. S.A.It., 73 N. 1, 213.

[38] Gaudenzi S., Giovannelli, F., , M., Corradini, M., Lombardi, R.: 2011, A\&A 525, 147.

[39] Giovannelli, F.: 1991, in Frontier Objects in Astrophysics and Particle Physics, F. Giovannelli \& G. Mannocchi (eds.), Italian Physical Society, Editrice Compositori, Bologna, Italy, 28, 3.

[40] Giovannelli, F.: 1996, in Multifrequency Behaviour of High Energy Cosmic Sources, F. Giovannelli \& L. Sabau-Graziati (eds.), Mem. S.A.It., 67, 401.

[41] Giovannelli, F.: 2008, ChJA\&A 8 Suppl., 237.

[42] Giovannelli, F., Gaudenzi, S., Lombardi, R., Bartolini, C., Guarnieri, A., Piccioni, A.: 1985, in Multifrequency Behaviour of Galactic Accreting Sources, F. Giovannelli (ed.), SIDEREA, Roma, Italy, p. 37.

[43] Giovannelli, F., Gonzalez Martinez-Pais, I., Gaudenzi, S., Lombardi, R., Claudi, R.U.: 1990, Ap\&SS 169, 125.

[44] Giovannelli, F., Martinez-Pais, I.G.: 1991, SSR, 56,313 .

[45] Giovannelli, F., Sabau-Graziati, L.: 1998, in Ultraviolet Astrophysics Beyond the IUE Final Archive, W. Wamstecker \& R.Gonzalez-Riestra (eds.), ESA SP-413, 419.

[46] Giovannelli, F., Sabau-Graziati, L.: 1999, in Multifrequency Behaviour of High Energy Cosmic Sources, F. Giovannelli \& L. Sabau-Graziati (eds.), Mem. S.A.It., 70, 987.

[47] Giovannelli, F., Sabau-Graziati, L.: 2004, SSR, 112,1 .

[48] Giovannelli, F., Sabau-Graziati, L.: 2012a, in The Golden Age of Cataclysmic Variables and Related Objects, F. Giovannelli \& L. Sabau-Graziati (eds.), Mem. S.A.It., 83 N. 2, 698.

[49] Giovannelli, F., Sabau-Graziati, L.: 2012b, in Second Workshop on Robotic Autonomous Observatories, Bull. Astr. Soc. India (in press).

[50] Giovannelli, F., Sabau-Graziati, L.: 2012c, Acta Polytechnica, 52 N. 1, 11.

[51] Godon, P., Sion, E.M.: 2002, ApJ, 566, 1084.
[52] Greggio, L., Renzini, A., Daddi, E.: 2008, arXiv: $0805.1512 \mathrm{v} 2$.

[53] Guy, J., Sullivan, M., Conley, A., Regnault, N., Astier, P., et al.: 2010, A\&A, 523, 7.

[54] Hack, M., la Dous, C.: 1993, in Cataclysmic Variables and Related Objects, M. Hack \& C. la Dous (eds.), NASA SP-507, 1.

[55] Hassall, B.J.M., Pringle, J.E., Schwarzenberg-Czerny, A., Wade, R.A., Whelan, J.A.J., Hill, P.W.: 1983, MNRAS, 203, 865. doi:10.1093/mnras/203.3.865

[56] Hassall, B.J.M., Pringle, J.E., Verbunt, F.: 1985, MNRAS, 216, 353.

doi:10.1093/mnras/216.2.353

[57] Heise, J., Mewe, R., Brinkman, A.C., Gronenschild, E.H.B.M., den Boggende, et al.: 1978, A\&A, 63, L1.

[58] Hellier, C.: 2001, Cataclysmic Variable Stars, Springer

[59] Horne, K., Marsh, T.R., Cheng, F.H., Hubeny, I., Lanz, T.: 1994, ApJ, 426, 294. doi:10.1086/174064

[60] Howell, D.A., Conley, A., Della Valle, M., Nugent, P., Perlmutter, S., et al.: 2009, arXiv0903, 1086H.

[61] Howell, S.B., Brinkworth, C., Hoard, D.W., Wachter, S., Harrison, T., et al.: 2006, ApJL 646, L65. doi:10.1086/506558

[62] Hoyle, F., Fowler, W.A.: 1960, ApJ, 132, 565. doi:10.1086/146963

[63] Hudec, R., Šimon, V., Munz, F., Gális, R.: 2008, ChJA\&AS, 8, 381.

[64] Iben, I., Jr., Tutukov, A.V.: 1984, ApJS, 54, 335.

[65] Isern, J., Hernanz, M., García-Berro, E.: 1993, in White Dwarfs: Advances in Observation and Theory, M.A. Barstow (ed.), Kluwer Academic Publ., Dordrecht, Holland, NATO ASI Ser., C403, 139 .

[66] Isern, J., Hernanz, M., Abia, C., José, J.: 1997, in Frontier Objects in Astrophysics and Particle Physics, F. Giovannelli \& G. Mannocchi (eds.), Italian Physical Society, Editrice Compositori, Bologna, Italy, 57, 113.

[67] de Jager, O.C., Meintjes, P.J., O’Donoghue, D., Robinson, E.L.: 1994, MNRAS, 267, 577. 
[68] Kilic, M., Brown, W.R., Allende Prieto, C., Agüeros, M.A.; Heinke, C., Kenyon, S.J.: 2011, ApJ, 727, 3.

[69] Knigge, C.: 2006, MNRAS, 373, 484.

[70] Knigge, C.: 2010, AIPC, 1314, 171.

[71] Knigge, C.: 2011, ASPC, 447, 3.

[72] Knigge, C., Baraffe, I., Patterson, J.: 2011, ApJS, 194,28

[73] Körding, E., Rupen, M., Knigge, C., Fender, R., Dhawan, V., et al.: 2008, Science 320, 1318.

[74] Kowalski, M., Rubin, D., Aldering, G, Agostinho, R.J., Amadon, A., et al.: 2008, ApJ, 686, 749. doi:10.1086/589937

[75] Lasota, J.-P., 2001: New Astron. Rev., 45, 449. doi:10.1016/S1387-6473(01)00112-9

[76] Lipunov, V.M.: 1987, Ap\&SS, 132, 1. doi:10.1007/BF00637779

[77] Lipunov, V.M.: 1991, in Frontier Objects in Astrophysics and Particle Physics, F. Giovannelli \& G. Mannocchi (eds.), Italian Physical Society, Editrice Compositori, Bologna, Italy, 28, 29.

[78] Long, K.S., Mauche, C.W., Raymond, J.C., Szkody, P., Mattei, J.A.: 1996, ApJ, 469, 841 doi:10.1086/177832

[79] Martinez-Pais, I.G., Giovannelli, F., Gaudenzi, S, Rossi, C.: 1996, A\&A, 308, 833.

[80] de Martino, D.: 1998, in Ultraviolet Astrophysics Beyond the IUE Final Archive, W. Wamstecker \& R.Gonzalez-Riestra (eds.), ESA SP-413, 387.

[81] de Martino, D.: 1999, in UV Astronomy in Italy, 1999, L. Buson \& D. de Martino (eds.), Mem. S.A.It., $70,547$.

[82] Mauche, C.W.: 1996, in Cataclysmic Variables and Related Objects, A.Evans \& J.H. Wood (eds.), IAU Coll. N. 158, Kluwer Academic Publishers, p. 243.

[83] Mauche, C.W.: 1998, in Wild Stars in the Old West S. Howell, E. Kuulkers \& C. Woodward (eds.), ASP Conf., 137, 113.

[84] Mauche, C.W., Wade, R.A., Polidan, R.S., van der Woerd, H., Paerels, F.: 1991, ApJ, 372, 659. doi:10.1086/170008

[85] Mauche, C.W., Raymond, J.C., Mattei, J.A.: 1995, ApJ, 446, 842. doi:10.1086/175842
[86] Meintjes, P.J., Raubenheimer, B.C., de Jager, O.C., Brink, C., Nel, H.I., et al.: 1992, ApJ, 401, 325. doi:10.1086/172063

[87] Meyer, F., Meyer-Hofmeister, E.: 1981, A\&A, 104, L10.

[88] Meyer, F., Meyer-Hofmeister, E.: 1982, A\&A, 106,34 .

[89] Meyer, F., Meyer-Hofmeister, E.: 1983, A\&A, 128,420 .

[90] Mikołajewska, J.: 2011, in Binary Paths in Type Ia Supernovae Explosions, R. Di Stefano \& M. Orio (eds.), IAU Symp. No. 281 (in press). 2011, arXiv: $1110.1847 \mathrm{v} 1$.

[91] Mitrofanov, I.G.: 1978, Sov. Astron. Lett., 4, 119.

[92] Mitrofanov, I.G.: 1980, in Close Binary Stars: Observations and Interpretations, D.M. Popper \& R.K. Ulrich (eds.), D. Reidel Publ. Co., Dordrecht, Holland, p. 431.

[93] Mouchet, M., Bonnet-Bidaud, J.M., de Martino, D.: 1998, in Ultraviolet Astrophysics, Beyond the IUE Final Archive, W. Wamstecker \& R. González-Riestra, (eds.), ESA Publication Division, ESTEC, Nordwijk, The Netherlands, ESA SP-413, 431.

[94] Nelemans, G.: 2005, ASPC, 330, 27.

[95] Norton, A.J., Beardmore, A.P., Allan, A., Hellier, C.: 1999, A\&A, 347, 203.

[96] Norton, A.J., Somerscales, R.V., Wynn, G.A.: 2004, in Magnetic Cataclysmic Variables, S. Vrielmann \&M. Cropper (eds.), IAU Coll. 190, ASP Conf. Ser., 315, 216.

[97] Norton, A.J., Wynn, G.A., Somerscales, R.V.: 2004, ApJ, 614, 349.

[98] Osaki, Y.: 1996, PASP, 108, 390.

[99] Paczyński, B.: 1967, AcA, 17, 5.

[100] Patat, F., Chandra, P., Chevalier, R., Justham, S., Podsiadlowski, Ph., et al.: 2008, The Messenger, 131, 30.

[101] Patterson, J.: 1979, ApJ, 234, 978. doi:10.1086/157582

[102] Patterson, J.: 1984, ApJS, 54, 443. doi:10.1086/190940

[103] Patterson, J.: 1994, PASP, 106, 209. doi:10.1086/133375 
[104] Patterson, J.: 1998, PASP, 110, 1132. doi:10.1086/316233

[105] Patterson, J., Raymond, J.C., 1985, ApJ, 292, 550. doi:10.1086/163188

[106] Phillips, M.M.: 2005, ASPC, 342, 211.

[107] Phillips, M.M.: 2011, arXiv: 11.4463v1.

[108] Phillips, M.M., Lira, P., Hamuy, M., Maza, J.: 1999, AJ, 118, 1766.

[109] Polidan, R.S., Holberg, J.B.: 1984, Nature 309, 528. doi:10.1038/309528a0

[110] Polidan, R.S., Holberg, J.B.: 1987, MNRAS, 309,528 .

[111] Pretorius, M.L., Warner, B., Woudt, P.A.: 2006, MNRAS, 368, 361. doi:10.1111/j.1365-2966.2006.10123.x

[112] Pringle, J.E., Wade, R.A. (eds.): 1985, Interacting Binary Stars, Cambridge Univ. Press.

[113] Rappaport, S., Cash, W., Doxsey, R., McClintock, J., Moore, G.: 1974, ApJ, 187, L5. doi:10.1086/181378

[114] Rappaport, S., Verbunt, F., Joss, P.C.: 1983, ApJ, 275, 713.

[115] Ricketts, M.J., King, A.R., Raine, D.J.: 1979, MNRAS, 186, 233.

[116] Reimer, T.W., Welsh, W.F., Mukai, K., Ringwald, F.A.: 2008, ApJ, 678, 376.

[117] Ritter, H.: 1992, in The Astronomy and Astrophysics Encyclopedia, Cambridge University Press, Cambridge, UK, p. 61.

[118] Ritter, H., Kolb, U.: 1998, A\&AS, 129, 83.

[119] Robinson, E.L.: 1976, ARA\&A, 14, 119.

[120] Rodriguez-Gil, P.: 2003, Ph.D. Thesis, La Laguna University, Spain.

[121] Rodríguez-Gil, P., Gänsicke, B.T., Hagen, H.-J., Araujo-Betancor, S., Aungwerojwit, A., et al.: 2007, MNRAS, 377, 1747. doi:10.1111/j.1365-2966.2007.11743.x

[122] Schaefer, B.E.: 2010, ApJS, 187, 275. doi:10.1088/0067-0049/187/2/275

[123] Schaefer, B.E.: 2011, ApJ, 742, 112. doi:10.1088/0004-637X/742/2/112
[124] Seitter, W.C..: 1990, in Physics of Classical Novae, A. Cassatella \& R. Viotti (eds.), Springer-Verlag, Lecture Notes in Physics, 369, 79.

[125] Shakura, N.I., Sunyaev, R.A.: 1973, A\&A, 24, 337.

[126] Šimon, V., Hudec, R., Munz, F., Štrobl, J.: 2006, ChJA\&A, 6 Suppl. 1, 149.

[127] Sion, E.M.: 1986, PASP, 98, 821. doi:10.1086/131831

[128] Sion, E.M.: 1991, AJ, 102, 295.

[129] Smak, J.: 1967, AcA, 17, 3.

[130] Smak, J.: 1984a, AcA, 34, 161.

[131] Smak, J.: 1984b, PASP, 96, 5. doi:10.1086/131295

[132] Smak, J.: 1985a, in Multifrequency Behaviour of Galactic Accreting Sources, F. Giovannelli (ed.), SIDEREA, Roma, Italy, p. 3.

[133] Smak, J.: 1985b, in Multifrequency Behaviour of Galactic Accreting Sources, F. Giovannelli (ed.), SIDEREA, Roma, Italy, p. 17.

[134] Smak, J.: 2002, in Multifrequency Behaviour of High Energy Cosmic Sources, F. Giovannelli \& L. Sabau-Graziati (eds.), Mem. S.A.It., 73 N. 1, 206.

[135] Stril, A., Cahn, R.N., Linder, E.V.: 2010, MNRAS, 404, 239.

[136] Surina, F., Bode, M.F., Darnley, M.J: 2011, arXiv: $1111.5524 \mathrm{v} 1$.

[137] Szkody, P.: 1998, in Ultraviolet Astrophysics, Beyond the IUE Final Archive, W. Wamstecker \& R. González-Riestra, (eds.), ESA Publication Division, ESTEC, Nordwijk, The Netherlands, ESA SP-413, 381.

[138] Terada, Y., Ishida, M., Bamba, A., Mukai, K., Hayashi, T., Harayama, A.: 2010, ApJ, 721, 1908.

[139] Townsley, D.M., Bildsten, L.: 2005, ApJ, 628, 395.

[140] Urban, J.A., Sion, E.M.: 2006, ApJ, 642, 1029. doi:10.1086/501430

[141] Verbunt, F.: 1987, A\&AS, 71, 339.

[142] Verbunt, F., Hassall, B.M.J., Pringle, J.E., Warner, B., Marang, F.: 1987, MNRAS, 225, 113. doi:10.1093/mnras/225.1.113

[143] Verbunt, F., Bunk, W.H., Ritter, H., Pfeffermann, E.: 1997, A\&A, 327, 602. 
[144] Voss, R., Nelemans, G.: 2008, Nature, 451, 802. doi:10.1038/nature06602

[145] Vrielmann, S., Cropper, M. (eds.): 2004, in Magnetic Cataclysmic Variables, IAU Colloquium 190, ASP Conf. Proc. Vol. 315. San Francisco: Astronomical Society of the Pacific.

[146] Warner, B.: 1976, The Observatory, 96, 49.

[147] Warner, B.: 1987, MNRAS, 227, 23. doi:10.1093/mnras/227.1.23

[148] Warner, B.: 1995a, Cataclysmic Variable Stars, Cambridge Astrophysics Series, 28, Cambridge University Press. doi:10.1017/CB09780511586491

[149] Warner, B.: 1995b, in Magnetic cataclysmic variables, D.A.H. Buckley \& B. Warner (eds.), ASP Conf. Ser., 85, 3.

[150] Warner, B.: 1995c, Ap\&SS, 225, 249. doi:10.1007/BF00613240

[151] Warner, B.: 1996, Ap\&SS 241, 263. doi:10.1007/BF00645229

[152] Warner, B.: 2001, in Small Telescope Astronomy on Global Scales, W.-P. Chen, C. Lemme, \& B. Paczynski (eds.), IAU Coll. 183, ASP Conf. Ser., 246, 159.

[153] Warner, B.: 2002, in Classical Nova Explosions, AIP Conf. Proc., 637, 3. doi:10.1063/1.1518170

[154] Warner, B.: 2004, PASP, 116, 115. doi:10.1086/381742

[155] Warner, B., Wickramasinghe, D.T.: 1991, MNRAS, 248, 370.

[156] Warner, B., Woudt, P.A.: 2005, in The Astrophysics of Cataclysmic Variables and Related Objects, J.-M. Hameury \& J.-P. Lasota (eds.), ASP Conf. Ser., 330, 227.
[157] Watson, M.G., King, A.R., Heise, J.: 1985, SSR, $40,127$.

[158] Webbink, R.F.: 1984, ApJ, 277, 355.

[159] Wheatley, P.J., Mauche, C.W., Mattei, J.A.: 2003, MNRAS, 345, 49.

[160] Whelan, J., Iben, I. Jr.: 1973, ApJ, 186, 1007.

[161] Willems, B., Kolb, U., Sandquist, E.L., Taam, R.E., Dubus, G.: 2005, ApJ, 635, 1263.

[162] Willems, B., Taam, R.E, Kolb, U., Dubus, G., Sandquist, E.L.: 2007, ApJ, 657, 465.

[163] Witham, A.R., Knigge, C., Aungwerojwit, A., Drew, J.E., Gänsicke, B.T., et al.: 2007, MNRAS, $382,1158$.

[164] Wood, J.H., Horne, K., Berriman, G., Wade, R.A.: 1989, ApJ, 341, 974.

[165] Woudt, P.A., Warner, B.: 2003, MNRAS, 340, 101.

[166] Woudt, P.A., Warner, B., Pretorius, M.L.: 2004, MNRAS, 351, 1015.

[167] Woudt, P.A., Warner, B., Spark, M.: 2005, MNRAS, 364, 107.

[168] Wynn, G.A.: 2000, New Astr. Rev., 44, 75.

[169] Wynn, G.A., King, A.R., Horne, K.: 1997, MNRAS, 286, 436.

[170] Yaron, O., Prialnik, D., Shara, M.M., Kovetz, A.: 2005, ApJ, 623, 398.

[171] Yoon, S.-C., Podsiadlowski, Ph., Rosswog, S.: 2007, MNRAS, 380, 933.

[172] Zhang, E., Robinson, E.L., Stiening, R.F., Horne, K.: 1995, ApJ, 454, 447. 